\title{
Intra-arterial anaesthetics for pain control in arterial embolisation procedures: a systematic review and meta-analysis
}

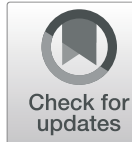

Taha Hanif Shiwani ${ }^{1^{*}}$ (D) and Hunain Shiwani ${ }^{2}$

\begin{abstract}
Purpose: A systematic review to determine the effectiveness of intra-arterial anaesthetics on post- operative pain and opioid analgesia requirements in arterial embolisation procedures.

Materials and methods: A systematic review of the literature was performed (Embase, PubMed, MEDLINE and the Cochrane Library) from inception to 10th August 2020. Randomised controlled trials (RCTs) and cohort studies that utilised intra-arterial anaesthesia during an embolisation procedure for the purposes of pain control were included. Eligibility was assessed by two investigators independently.

Results: Eight hundred fifty-nine candidate articles were identified, and 9 studies met the inclusion criteria (6 RCTs and 3 retrospective cohort studies). Four studies were of hepatic chemoembolisation and 5 were of uterine artery embolisation.

Five hundred twenty-nine patients were treated in total. All studies used lidocaine as the anaesthetic with doses ranging from 20 to $200 \mathrm{mg}$, and the anaesthetic was delivered varyingly before, during or after embolisation. Pain intensity was converted to a numeric scale from 0 to 10, and opioid doses were converted to milligram morphine equivalent doses. A random-effects meta-analysis model was used to analyse the results of RCTs, and the results of cohort studies were summarised with a narrative synthesis. The meta-analyses suggested that pain scores were reduced by a mean of $1.02(95 \% \mathrm{Cl}-2.34$ to $0.30 ; p=0.13$ ) and opioid doses were reduced by a mean of $7.35 \mathrm{mg}$ $(95 \% \mathrm{Cl},-14.77,0.06 ; p=0.05)$ in the intervention group however neither finding was statistically significant. No serious adverse events were reported.

Conclusion: Intra-arterial anaesthetic may slightly reduce pain intensity and post-operative opioid consumption following embolisation, however the results are not statistically significant. There is very limited data available on the effect of anaesthetic on length of hospital admission. Whilst no serious adverse events were reported, there are some concerns regarding the effect of lidocaine on the technical success of embolisation procedures that preclude our recommendation for routine use in embolisation procedures.

High quality randomised controlled trials are required to elucidate the dose-response effect of lidocaine on opioid consumption and pain following embolisation, particularly in the first few hours post-operatively, as well as effects on duration of hospital stay.
\end{abstract}

Keywords: Systematic review, Meta-analysis, Embolisation, Embolization, Arterial, Intraarterial, Anaesthetic, Anaesthesia, Pain control, Pain management, Interventional radiology

\footnotetext{
* Correspondence: shiwanitaha@gmail.com

${ }^{1}$ Imperial College London, London, UK

Full list of author information is available at the end of the article
}

\section{Springer Open}

(c) The Author(s). 2021 Open Access This article is licensed under a Creative Commons Attribution 4.0 International License, which permits use, sharing, adaptation, distribution and reproduction in any medium or format, as long as you give appropriate credit to the original author(s) and the source, provide a link to the Creative Commons licence, and indicate if changes were made. The images or other third party material in this article are included in the article's Creative Commons licence, unless indicated otherwise in a credit line to the material. If material is not included in the article's Creative Commons licence and your intended use is not permitted by statutory regulation or exceeds the permitted use, you will need to obtain permission directly from the copyright holder. To view a copy of this licence, visit http://creativecommons.org/licenses/by/4.0/. 


\section{Background}

Arterial embolisation is currently best practice for a variety of disease processes with expanding and increasingly novel indications. However, embolisation procedures can often be complicated by significant post-operative pain due to the ischaemia and subsequent inflammation they induce in downstream tissues (Spencer et al. 2013).

Pain post embolisation is most clearly documented in the context of trans-arterial chemoembolisation (TACE) for HCC (Mason et al. 2015) and uterine fibroid embolisation (UFE) (Edwards et al. 2007). Post-operative pain has been strongly implicated in increasing a patient's length of hospital stay and risk of overnight and recurrent admission following these procedures (Leung et al. 2001) (Spencer et al. 2013).

Protocols for pain control following arterial embolisation vary drastically between procedures and between institutions, however, most rely on traditional methods of patient controlled opioid analgesia (PCA) or intravenous (IV) opioid medication pre/post-operatively before transitioning patients on to oral opioids, non-steroidal anti-inflammatory drugs and/or paracetamol. Whilst effective, opioid medications are associated with significant adverse effects, most commonly nausea/vomiting and cognitive dysfunction (Swegle and Logemann 2006). Consequently, alternative measures for pain control have been proposed including local nerve blocks (e.g superior hypogastric nerve block for UFE) (Spencer et al. 2013) and intra-arterial administration of anaesthetic in the peri-procedural period.

The latter has been reported as a simple and transferrable intervention to reduce pain scores, reliance on opioid medication and recovery time following embolisation, however, there is no consensus in the literature regarding its safety and effectiveness. The potential mechanism of action is also unclear, with a suggestion that anaesthetics may diffuse into the arterial wall and exert a prolonged effect on local tissues following embolisation (Hartnell et al. 1999). This is thought to be due to reduced metabolism of the anaesthetic following occlusion of the local blood supply as well as decreased washout of the drug.

In this review, we aimed to systematically review and analyse the effectiveness of intra-arterial anaesthetics on reducing post-operative pain and opioid analgesia requirements in arterial embolisation procedures, with a secondary aim to determine the effect on length of hospital stay.

\section{Main text Methods}

This study was registered on PROSPERO (CRD42020176020). A systematic review of the literature was performed (Embase, PubMed, MEDLINE and the
Cochrane Library) from inception to 10th August 2020. A pre-determined search strategy was used with appropriate key words and Boolean operators (Additional file 1: Appendix 1).

Records obtained by the search strategy were aggregated into RefWorks. Duplicate records were identified and discarded. The abstracts and titles of all records were screened to exclude conference papers, reviews, case-series, articles that were not published in English, and articles that did not make reference to intra-arterial delivery of an anaesthetic for pain control in an embolisation procedure. The full texts of remaining articles were then reviewed against pre-defined eligibility criteria. Randomised trials and non-randomised prospective or retrospective cohort studies were included if they reported either a pain score or post-procedural analgesia requirements up to $48 \mathrm{~h}$ after an embolisation procedure. The following studies were excluded: case reports or case-series, those including children $(<16$ yrs. of age), those not using an anaesthetic, those without a control arm (either placebo or no treatment), and those without a full-text article published in English.

The screening and selection processes were conducted independently by two investigators including a senior interventional radiology registrar.

\section{Data extraction}

The following data was obtained from the published report of each study and collated into a Microsoft Excel spreadsheet: study design; demographic details; indication for embolisation, method of embolisation; timing, type and dose of anaesthetic delivered; details of control arm; post-procedural pain score and/or analgesia requirements; length of hospital stay (where recorded); and complications/adverse events.

Post-procedural pain scores were standardised to a numerical rating scale (NRS) score from 0 to 10. Studies that did not include quantitative pain scores were excluded from the meta-analysis. A VAS score between 0 to $10 \mathrm{~cm}$ was considered to be equivalent to a numerical rating scale (NRS) score of 0 to 10 as they have been shown to be strongly correlated (Downie et al. 1978). Where pain intensity was measured using a visual analogue scale (VAS) of 0 to $100 \mathrm{~cm}$, the VAS scores were converted to a score between 0 to 10 by dividing by 10 .

Similarly, where required, post-procedural analgesia requirements were converted to milligram morphine equivalent doses using conversion ratios described in the literature. These included the following: 10:1 for IV meperidine: IV morphine (Pereira et al. 2001), 1:1 for IV nalbuphine: IV morphine (Zeng et al. 2015), 1:5 for IV hydromophone: IV morphine (Patanwala et al. 2007). 


\section{Risk of bias assessment}

Each study included in the review was subjected to a risk of bias assessment. These were conducted independently by two investigators, with disagreements being resolved through discussion.

The risk of bias in randomised controlled trials (RCTs) was assessed using the Risk of Bias 2 (RoB 2) Tool developed by the Cochrane Collaboration (Sterne et al. 2019). This assesses the risk of biases in the following domains: 1) bias arising from the randomization process; 2) bias due to deviations from intended interventions; 3) bias due to missing outcome data; 4) bias in measurement of the outcome; 5) bias in selection of the reported result. Using the RoB 2 Tool, a judgement of "low risk", "some concerns" or "high risk" was made for the risk of bias in each domain, allowing an overall risk of bias to be generated for each study using the tools algorithm.

The risk of bias in non-randomised cohort studies was assessed using the Newcastle-Ottawa Scale (NOS) (Wells et al. 2012). This considers the following study characteristics: 1) participant selection; 2) comparability of cohorts 3) outcome measurement. For ease of presentation, the NOS score was translated to a quality judgement of "good", "fair" or "poor" as outlined by the Agency for Healthcare Research and Quality (AHRQ) using AHRQ conversion thresholds (Agency for Healthcare Research and Quality 2014).

\section{Data analysis \& synthesis}

Results for all outcomes were expressed as means +/standard deviations where possible. The formulas recommended by the Cochrane Handbook for Systematic Reviews of Interventions (Higgins et al. 2019) were used to calculate the standard deviation from confidence intervals or standard errors where necessary (Additional file 1: Appendix 2). If studies had more than one treatment arm, the groups were combined as recommended by the Cochrane Handbook (Higgins et al. 2019) (Additional file 1: Appendix 3).

The results of the RCTs were synthesised using a random-effects meta-analysis model to generate pooled mean differences for each primary outcome measurement, and a $\mathrm{z}$-test was used to determine generate a $p$ value and determine statistical significance. A randomeffects model was used as there was significant methodological and clinical heterogeneity in included studies. Mean difference was felt to be an appropriate summary statistic as all pain scores and opioid requirements were converted to the same scales. A forest plot was generated for each primary outcome measurement, including post-embolisation pain scores and opioid requirements. Where multiple pain scores were provided in a study, the mean pain score in the first $24 \mathrm{~h}$ post-procedure was selected for the forest plot, as it was the most consistently measured statistic and clinically influences the need for overnight hospital admission. Similarly, where multiple values for post-procedural opioid requirements were provided, the total dose of opioid administered post-procedure was included in the forest plot as it was the most consistently measured statistic. Statistical heterogeneity for all outcomes was quantified using the $\mathrm{I}^{2}$ statistic as described in the Cochrane Handbook (Higgins et al. 2019), and all analyses were conducted using RevMan 5 software. As only a small number of studies were ultimately included in the analyses, subgroup analyses and sensitivity analyses were not performed.

The results of the cohort studies were summarised separately using a narrative synthesis effect due to the variability in their outcome effect measures and use of statistical tests.

This systematic review was conducted using the guidelines documented in the 2009 PRISMA checklist (Moher et al. 2009).

\section{Results \\ Search results}

The search strategy yielded 1149 records. Of those, 290 were discarded as duplicates and 844 were discarded upon screening of their abstract and title. The full text of the remaining 15 records were then reviewed, revealing 9 eligible studies. This is summarised in detail using the PRISMA flowchart in Fig. 1.

\section{Characteristics of included studies}

Nine studies reported a total of 548 embolisation procedures (316 intervention and 232 controls) in 529 participants.

Six studies were RCTs (Abusedera et al. 2014; Duvnjak and Andersen 2020; Keyoung et al. 2001; Lee et al. 2001; Noel-Lamy et al. 2017; Zhan et al. 2005) and three were cohort studies: two of mixed-design (control group studied retrospectively, intervention group studied prospectively), (Molgaard et al. 1990; Hartnell et al. 1999), and one retrospective cohort study (Katsumori et al. 2020).

Five studies investigated UFE (Duvnjak and Andersen 2020; Keyoung et al. 2001; Katsumori et al. 2020; NoelLamy et al. 2017; Zhan et al. 2005), two studies investigated hepatic TACE for the treatment of HCC alone (Abusedera et al. 2014; Lee et al. 2001) and two studies investigated hepatic TACE for HCC and metastatic disease (Molgaard et al. 1990, Hartnell et al. 1999). This is summarised in Table 1.

Lidocaine was the anaesthetic of choice in all studies with doses ranging from 20 to $200 \mathrm{mg}$, and concentrations ranging from $0.67 \%$ to $10 \%$. It was administered varyingly before, during or after embolisation.

The detailed characteristics of all included studies are summarised in Additional file 2: Appendix 4. 


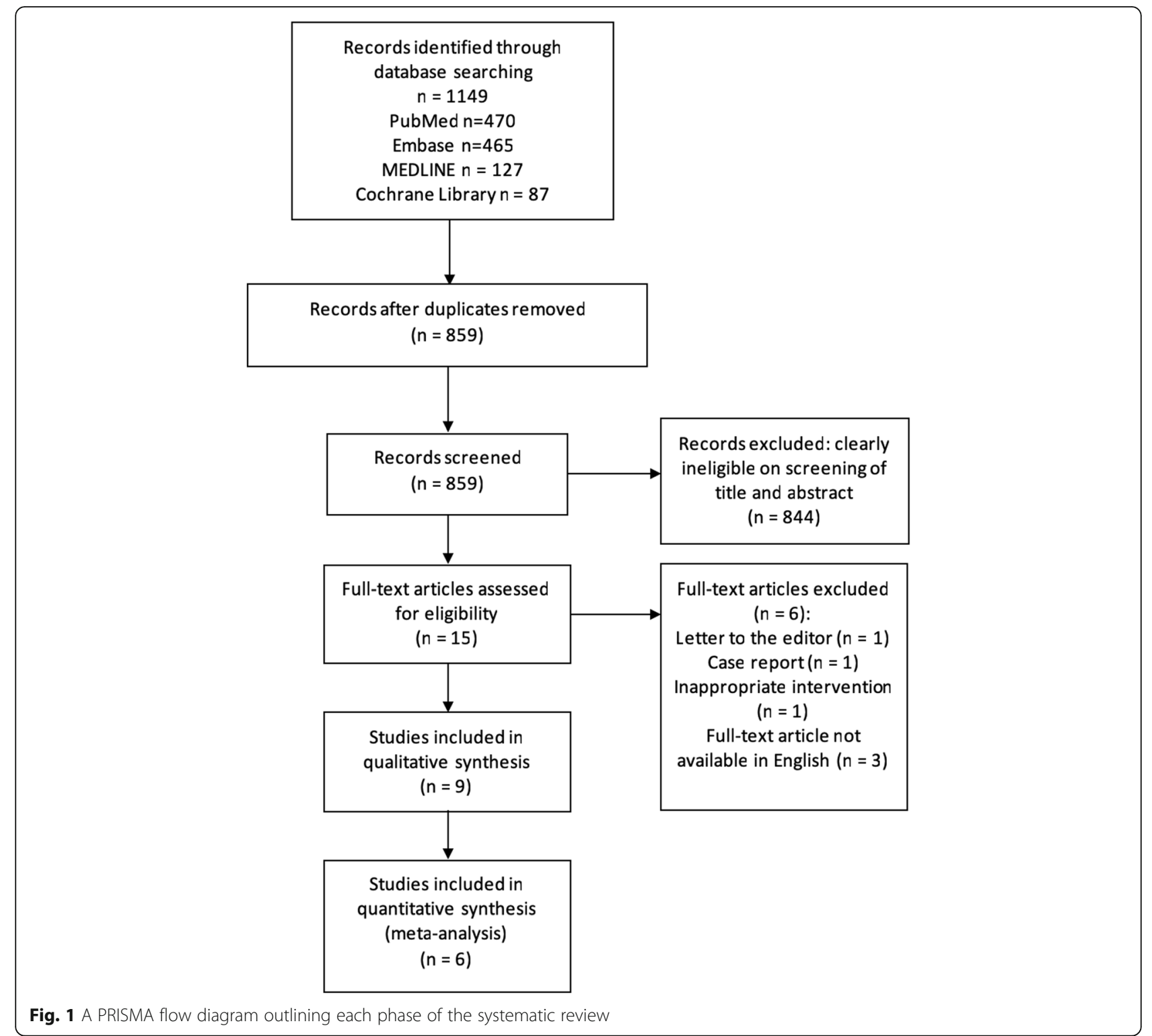

\section{Pain scores}

\section{$R C T$ s and meta-analysis}

Data from five RCTs $(n=267)$ showed that pain scores were not significantly lower in those that received anaesthetic. The pooled mean difference (PMD) in pain scores was 1.02 (95\% CI: -2.34 to $0.30 ; p=0.13$ ) when comparing the study group $(n=167)$ to the control group $(n=100)$. The $\mathrm{I}^{2}$ statistic was 84\%. The forest plot is presented in Fig. 2.

An intention-to-treat (ITT) analysis was not reported by Duvnjak and Andersen (2020), and so the perprotocol results from the study were used for the metaanalysis.

Table 1 A table summarising the studies included in this review and the embolization procedures that they investigated

\begin{tabular}{lll}
\hline & Randomised Controlled Trials (RCTs) & Cohort Studies \\
\hline Uterine fibroid embolization (UFE) & Duvnjak and Andersen (2020) & Katsumori et al. (2020) \\
& Keyoung et al. (2001) & \\
& Noel-Lamy et al. (2017) & Hartnell et al. (1999) \\
Hepatic transarterial chemoembolization (TACE) & Zhan et al. (2005) & Molgaard et al. (1990) \\
\hline
\end{tabular}




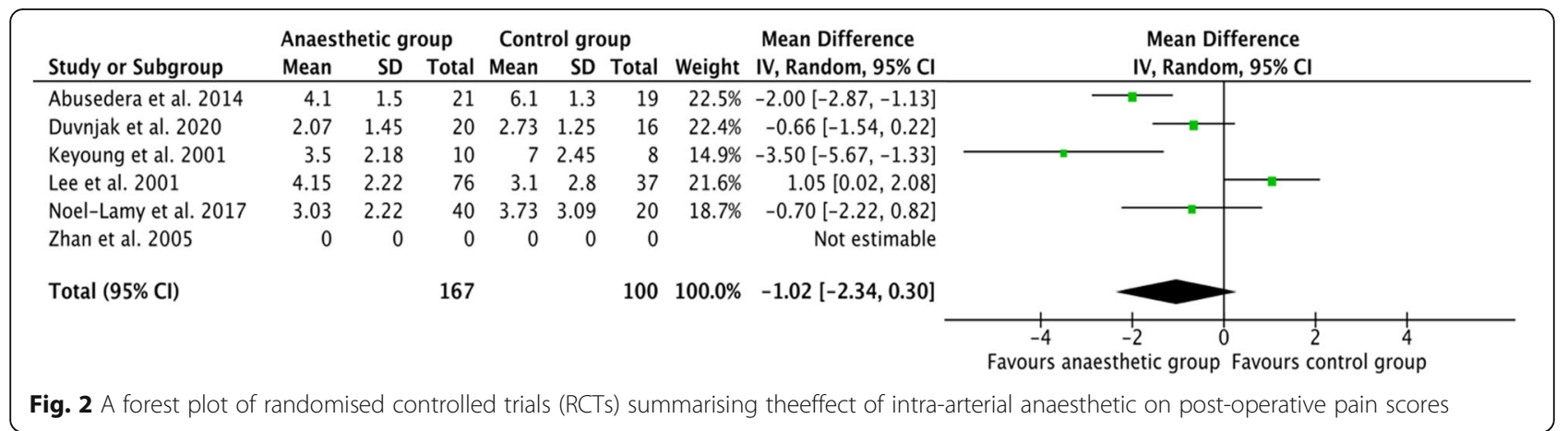

Notably, in those RCTs that reported pain scores at several time points, statistically significant reductions in pain scores were noted at $2 \mathrm{~h}$ by Duvnjak and Andersen (2020) (PMD: $-1.82, p=0.013$ ) and at $4 \mathrm{~h}$ by Noel-Lamy et al. (2017) (PMD: $-2.71, p<0.001$ ).

Zhan et al. (2005) despite being an RCT, used a qualitative 6-point pain rating scale and was therefore excluded from the meta-analysis of pain scores. A statistically significant reduction in pain $(p<0.01)$ was seen within the first $48 \mathrm{~h}$ post-procedure in the study group $(n=23)$ compared to the control group $(n=23)$.

The full results are presented in Additional File 2: Appendix 5.

\section{Cohort studies}

One cohort study (Katsumori et al. 2020) reported pain scores over several time periods between 0 and $24 \mathrm{~h}$ post-procedure. However, no statistically significant differences in VAS scores were noted at any time point.

The full results are presented in Additional File 2: Appendix 6.

\section{Post-procedural opioid requirements RCTs and meta-analysis}

Data from five RCTs $(n=267)$ showed that postprocedural opioid requirements were not significantly lower in those that received anaesthetic. The pooled mean difference was $-7.35 \mathrm{mg}$ morphine equivalent (95\% CI: $-14.77,0.06 ; p=0.05$ ) when comparing the study group $(n=167)$ to the control group $(n=100)$. The $\mathrm{I}^{2}$ statistic was $93 \%$. The forest plot is presented in Fig. 3.

An intention-to-treat (ITT) analysis was not reported by Duvnjak and Andersen (2020), and so the perprotocol results were used in the analysis.

The full results are presented in Additional File 2: Appendix 7.

\section{Cohort studies}

Three cohort studies reported post-procedural opioid requirements (Hartnell et al. 1999; Katsumori et al. 2020; Molgaard et al. 1990). Although two out of the three studies found a statistically significant reduction in postoperative opioid requirements, the result should be interpreted with great caution due to the limited number of studies and high risk of bias in the included studies.

Hartnell et al. (1999) recorded the total dose of opioid used in the first $24 \mathrm{~h}$ following the procedure. There was a statistically significant decrease in opioid use (mean difference $-26.75 \mathrm{mg}$ morphine equivalent, $p=0.0016$ ) when comparing the study group $(n=29)$ to the control group $(n=35)$.

Molgaard et al. (1990) reported the incidence of the requirement for a continuous morphine infusion following the embolisation procedure. There was a statistically significant decrease in the incidence of morphine infusion usage $(60 \%$ lower, $p<0.001)$ in the study group $(n=45)$ than in the control group $(n=20)$.

Katsumori et al. (2020) reported the total dose of opioid administered following the embolisation procedure, although opioids were used only in the first $12 \mathrm{~h}$ following the procedure. There was a non-statistically significant difference in morphine dose $(0.50 \mathrm{mg}$ morphine equivalent [95\% CI:-0.72 to 1.72], $p=0.42$ ) when comparing the study group $(n=50)$ to the control group $(n=50)$.

The full results are presented in Additional file 2: Appendix 8.

\section{Length of hospital stay \\ RCTs}

Only two RCTs recorded length of hospital stay (Abusedera et al. 2014; Noel-Lamy et al. 2017). Consequently, a meta-analysis was not deemed appropriate.

Neither study reported a statistically significant difference in the mean length of hospital stay. The mean differences in length of hospital stay were $-2.4 \mathrm{~h}$ (95\% CI: $-27.69,22.89 ; p=0.85$ ) (Abusedera et al. 2014) and - 


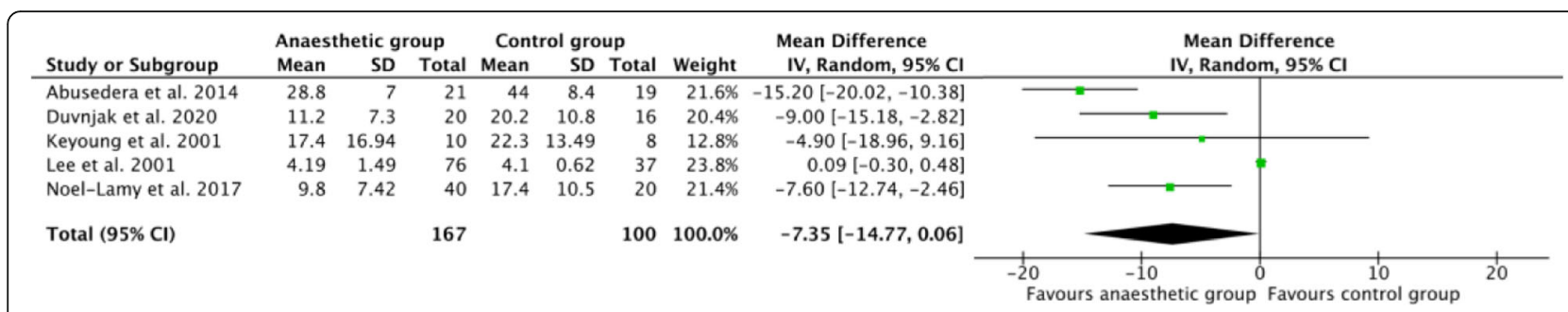

Fig. 3 A forest plot of randomised controlled trials (RCTs) summarising the effect of intra-arterial anaesthetic on post-operative opioid requirements

0.9 h (95\% CI: $-4.03,2.23 ; p=0.55)$ (Noel-Lamy et al. 2017) when comparing the study group to the control group.

The full results are presented in Additional file 2: Appendix 9 .

\section{Cohort studies}

One cohort study (Hartnell et al. 1999) recorded the mean length of hospital stay of patients admitted following hepatic TACE. There was a statistically significant decrease in mean length of hospital stay $(-14 \mathrm{~h}, p=$ $0.049)$ when comparing the study group $(n=29)$ to the control group $(n=35)$.

The full results are presented in Additional file 2: Appendix 10 .

\section{Complications \& adverse events}

Three studies reported some complications or adverse events (Keyoung et al. 2001; Lee et al. 2001; Noel-Lamy et al. 2017) associated with use of intra-arterial anaesthetic. A summary is presented in Table 2.
Keyoung et al. (2001) intended to recruit a total of 126 patients, however, the trial was concluded prematurely as 6 of 9 patients that had received $200 \mathrm{mg}$ lidocaine developed moderate to severe vasospasm that restricted flow for 10 to $20 \mathrm{~min}$. As a doserelated problem had been suspected, a final patient was given $100 \mathrm{mg}$ lidocaine, however they also developed significant vasospasm. This had not been noted in the control group.

Lee et al. (2001) reported a transient drop in blood pressure from $120 / 80 \mathrm{mmHg}$ to $90 / 60 \mathrm{mmHg}$ following lidocaine delivery, however, this was only recorded in 1 out of 76 patients that received lidocaine, and it resolved over $10 \mathrm{~min}$.

Noel-Lamy et al. (2017) reported complete leiomyoma infarction at 3-month follow up in just 38.9\% of patients who received lidocaine during UFE $(n=$ 20 ), compared to $77.8 \%$ in those who received lidocaine after UFE $(n=20)$, and $75 \%$ in the control group $(n=20)$. This difference was determined to be statistically significant $(p=0.045)$ using a KruksalWallis test.

Table 2 A table summarising the complications and adverse events of all included studies

\begin{tabular}{|c|c|c|}
\hline Study & Type of Study & Complications \& Adverse events \\
\hline Abusedera et al. 2014 & $\mathrm{RCT}$ & None reported. \\
\hline $\begin{array}{l}\text { Duvnjak and Andersen } \\
2020\end{array}$ & $\mathrm{RCT}$ & None reported. \\
\hline Hartnell et al. 1999 & $\begin{array}{l}\text { Observational (retrospective \& } \\
\text { prospective) }\end{array}$ & None reported. \\
\hline Katsumori et al. 2020 & Observational (retrospective) & None reported. \\
\hline Keyoung et al. 2001 & $\mathrm{RCT}$ & $\begin{array}{l}\text { Trial concluded prematurely. } 7 / 10 \text { of those receiving lidocaine had moderate } \\
\text { to severe vasospasm with restricted flow for } 10-20 \mathrm{~min} \text {. }\end{array}$ \\
\hline Lee et al. 2001 & $\mathrm{RCT}$ & $\begin{array}{l}\text { One patient had a transient }(\sim 10 \mathrm{~min}) \text { decrease in blood pressure } \\
\text { (from } 120 / 80 \mathrm{mmHg} \text { to } 90 / 60 \mathrm{mmHg} \text { ) after lidocaine injection. }\end{array}$ \\
\hline Molgaard et al. 1990 & $\begin{array}{l}\text { Observational (retrospective \& } \\
\text { prospective) }\end{array}$ & None reported. \\
\hline Noel-Lamy et al. 2017 & $\mathrm{RCT}$ & $\begin{array}{l}\text { Statistically significant reduction }(p=0.045) \text { in proportion of patients with } \\
\text { complete infarction of leiomyoma in those receiving lidocaine during } \\
\text { UFE }(38.9 \%)(n=18) \text { compared to those receiving lidocaine after UFE } \\
(77.8 \%)(n=18) \text { and control group }(75 \%)(n=20) \text {. }\end{array}$ \\
\hline Zhan et al. 2005 & $\mathrm{RCT}$ & None reported \\
\hline
\end{tabular}




\section{Risk of bias assessment}

A summary of the risk of bias assessment of RCTs using the RoB 2 tool is presented in Fig. 4. The risk of bias assessment was identical for both primary outcomes. Overall, 3 studies were judged to have a high risk of bias and 3 judged to have a low risk. Of the former, all 3 were at high risk of bias with regards to the measurement of primary outcomes due to a lack of blinding, and there were some concerns regarding the randomization processes as detailed methods were not reported. There were additional concerns noted regarding missing data in Duvnjak and Andersen (2020).

A summary of the quality assessment of cohort studies using the NOS tool is presented in Table 3. Two studies were judged to be of "poor" quality (Hartnell et al. 1999; Molgaard et al. 1990) primarily due to a lack of comparability between intervention and control groups, while the remaining study was judged to be of "good" quality (Katsumori et al. 2020).

\section{Discussion}

In the post-operative setting, a reduction in VAS score of $0.99 \mathrm{~cm}$ has been shown to be the minimum clinically significant difference (MCSD) that a patient would recognise as a decrease in pain intensity (Myles et al. 2017). The meta-analysis performed in this review suggested that mean pain scores are decreased by 1.02 (95\% CI -
2.34 to $0.30 ; p=0.13$ ) with the use of intra-arterial anaesthesia which suggests a clinically significant difference. However, as the results are not statistically significant, the results should be interpreted with a degree of caution.

Similarly, a dose-response relationship between opioid use and adverse events is well-documented, and even a $3 \mathrm{mg}$ morphine equivalent increase in opioid dose has been associated with an increase in opioid-related symptoms (Zhao et al. 2004). Therefore, the results of our meta-analyses that indicate a mean reduction in opioid dose of $7.35 \mathrm{mg}$ morphine equivalent (95\% CI: -14.77 , $0.06 ; p=0.05)$ with intra-arterial anaesthesia suggests a clinically significant difference. However, once again, given the heterogeneity between studies and lack of statistical significance of the results, this should be interpreted with a high level of caution.

The anaesthetic effect of lidocaine (the anaesthetic used by all included studies) derives from its action as a sodium channel blocker. It delays the depolarisation of neurones and thereby inhibits pain signalling (Beecham et al. 2020). The prevailing theory for the proposed analgesic action of lidocaine in embolisation procedures was first suggested by Hartnell et al. (1999); it was theorised that lidocaine would diffuse into the local arterial wall and provide prolonged anaesthesia due to reduced blood flow and washout of the agent following embolisation. In

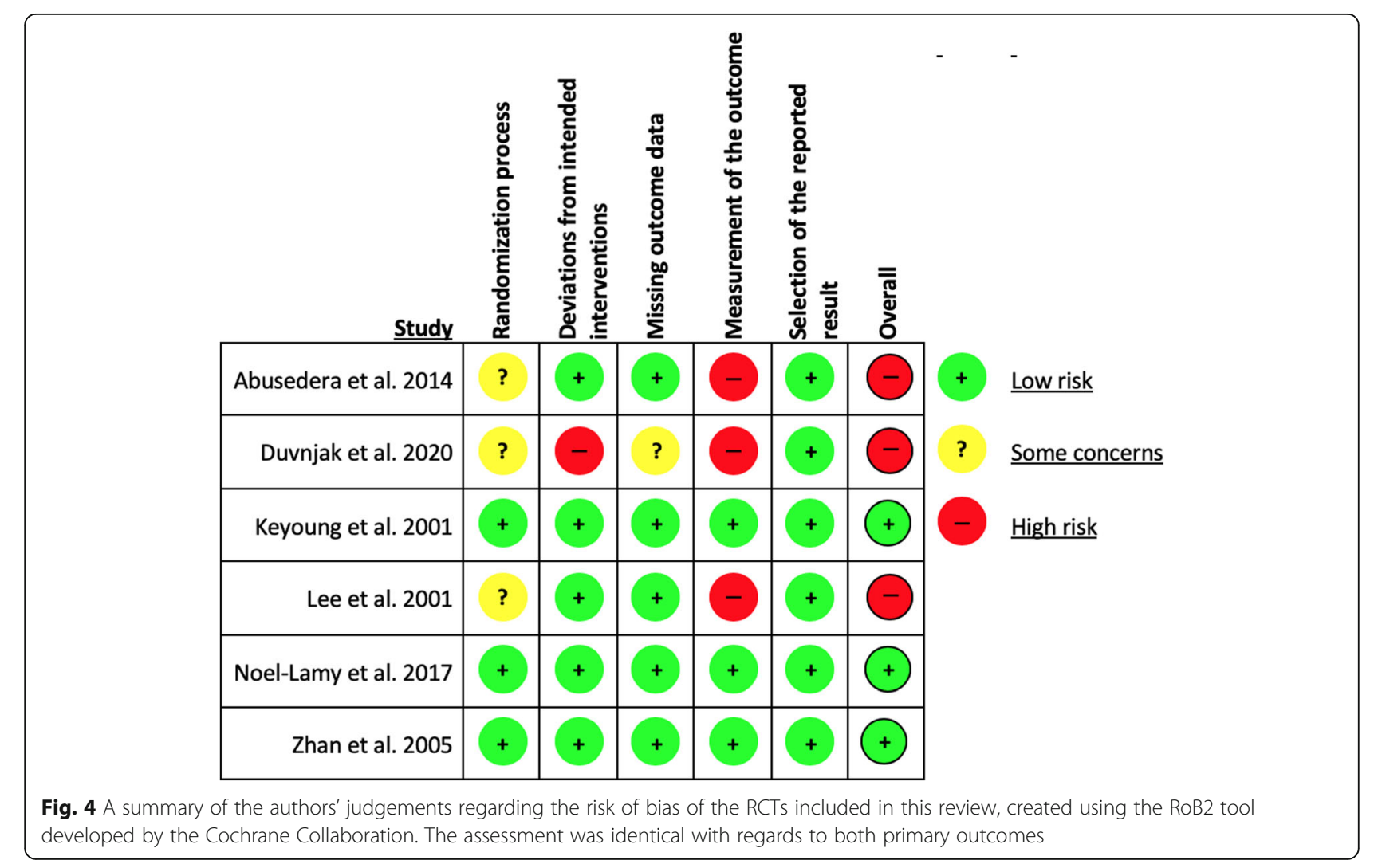


Table 3 A table summarising the Newcastle-Ottawa Quality Assessment Scale judgements made for the cohort studies included in this review

\begin{tabular}{|c|c|c|c|}
\hline & $\begin{array}{l}\text { Hartnell } \\
\text { et al. } 1999\end{array}$ & $\begin{array}{l}\text { Katsumori } \\
\text { et al. } 2020\end{array}$ & $\begin{array}{l}\text { Molgaard } \\
\text { et al. } 1990\end{array}$ \\
\hline \multicolumn{4}{|l|}{ Selection } \\
\hline Representativeness of exposed cohort & * & * & \\
\hline Selection of the non-exposed cohort & & * & \\
\hline Ascertainment of exposure & * & * & * \\
\hline Demonstration that outcomes of interest were not present at start of study & & & * \\
\hline \multicolumn{4}{|l|}{ Comparability } \\
\hline $\begin{array}{l}\text { Comparability of cohorts on the basis of the design or analysis controlled for confounders } \\
\text { (other factors incl. Patient demographics, severity of disease, embolisation procedure) }\end{array}$ & & ** & \\
\hline \multicolumn{4}{|l|}{ Outcome } \\
\hline Assessment of outcome & * & * & * \\
\hline Was follow-up long enough for outcomes to occur & * & * & * \\
\hline Adequacy of follow-up of cohorts & * & * & * \\
\hline Total Newcastle-Ottawa Score: & 5 & 8 & 5 \\
\hline AHRQ Judgement: & Poor quality & Good quality & Poor quality \\
\hline
\end{tabular}

As outlined by the Newcastle-Ottawa Scale, each study was awarded a maximum of one star for each item within the Selection and Outcome categories. A maximum of two stars was given for Comparability. The total number of stars awarded to each study was translated to a qualitative judgement using conversion thresholds developed by The Agency for Healthcare Research and Quality

addition, in vitro studies have shown that intra-arterial administration of lidocaine reduces the release of inflammatory mediators such as leukotriene B4 and interleukin-1 from granulocytes and mononuclear cells (Sinclair et al. 1993). Regarding TACE procedures specifically, lidocaine may also counteract the irritation caused to arteries by injection of a chemotherapeutic solution, as lidocaine has been shown to reduce pain caused by injection of contrast material during peripheral angiography (Widrich et al. 1977).

However, given that lidocaine has a half-life between 90 to $120 \mathrm{~min}$ (Beecham et al. 2020), it is unlikely that any analgesic effect would last up to $24 \mathrm{~h}$ after the procedure. This may explain why meta-analyses of mean pain scores over the first $24 \mathrm{~h}$ and total post-procedural opioid consumption led to results that were not statistically significant. Further study of the short-term effects of lidocaine is required.

Noel-Lamy et al. (2017) have also suggested that the injection of lidocaine after embolisation may simply lead to reflux of most of the anaesthetic, reducing its analgesic effect. Therefore, where treatment arms were combined in this analysis, the treatment effect may have been underestimated.

The studies that reported significant technical difficulties (Noel-Lamy et al. 2017; Keyoung et al. 2001) both used relatively high doses of lidocaine $(200 \mathrm{mg})$ and investigated UFE specifically. The difficulties observed were associated with vasospasm induced by lidocaine; Keyoung et al. (2001) observed vasospasm directly, whilst Noel-Lamy et al. (2017) theorised that complete infarction of leiomyomata was prevented by distal vasospasm. Lidocaine is typically known to produce vasodilation and has been used to relieve vasospasm in endovascular procedures (Ishihara et al. 2015), however, there may be a dose-related effect or an effect specific to uterine arteries, as an in-vitro study showed that uterine arteries obtained from hysterectomies underwent transient dose-dependent contractions when injected with lidocaine (Cibils 1976).

Similar to this review, a previous systematic review published in 2019 assessed various pain management protocols in uterine fibroid embolisation, including intra-arterial administration of anaesthetic (Saibudeen et al. 2019). It included 3 studies on UFE and concluded that intra-uterine drug administration provided slightly better pain control compared to other analgesic protocols whilst having no significant effect on the length of hospital stay. This review provides some supportive data regarding the analgesic effect of intra-arterial anaesthetic, and also assesses the effect on post-operative opioid consumption, whilst incorporating data from additional trials and observational studies investigating both UFE and hepatic TACE.

Larger, high-quality RCTs are required to determine the utility of intra-arterial anaesthetic, particularly with regards to: the optimal timing and dosage of administration; the immediate post-operative analgesic effects; the long-term effects on hospital stay and technical success of embolization; and the importance of covariates such as the type of embolization procedure, the volume of tissue infarction, and patient comorbidities. Future studies 
should also aspire to report pain scores in a standardised format such as with a visual analog scale (VAS) or numerical rating scale (NRS) and should aim to provide granular data on tissue type including size of treated pathology as this would influence the degree of tissue ischaemia.

\section{Limitations}

Individual studies often did not report or control for significant confounding factors such as patient demographics and pain or disease severity prior to embolisation. Relevant outcomes, such as length of hospital stay and post-operative nausea and vomiting were also reported inconsistently. When comparing studies, there was significant clinical heterogeneity with regards to: the method of embolisation; the dose, concentration and timing of lidocaine use; intra-operative analgesic protocols; post-operative non-opioid analgesic protocols; and the timing and method of primary outcome measurements. All of these factors may have affected the effect sizes, and this heterogeneity was likely reflected in the high $\mathrm{I}^{2}$ values noted in the meta-analyses. As a result, all the statistical tests that were conducted should be interpreted with caution, especially given the small number of studies (each with relatively few participants) and the high risk of bias within many of the studies, particularly the observational studies.

The general applicability of this review to all embolisation procedures is further limited by the fact that included studies only investigated UFE and hepatic TACE, with no data available for other types of embolisation procedures. The effectiveness of intra-arterial anaesthetic may be dependent upon the volume and type of tissue that is embolized, but a lack of granular data available currently precludes effective subgroup or covariate analyses.

\section{Conclusions}

Intra-arterial anaesthetic may slightly reduce pain intensity and post-operative opioid consumption following embolisation, however the results are not statistically significant. There is very limited data available on the effect of anaesthetic on length of hospital admission, and some concerns regarding the effect of lidocaine on the technical success of embolization procedures.

There is a general lack of high-quality studies, and significant clinical heterogeneity in existing studies. High quality randomised controlled trials are required to elucidate the dose-response effect of lidocaine on opioid consumption and pain following embolisation, particularly in the first few hours post-operatively.

\section{Supplementary Information}

The online version contains supplementary material available at https://doi. org/10.1186/s42155-020-00198-z.

Additional file 1: Appendix 1. Search strategy used for each database. Performed on 10th August. Appendix 2. Formula used to calculate standard deviation from confidence intervals or standard errors. Appendix 3. Formulae used to combine mean, standard deviation and sample sizes of studies containing 2 treatment arms.

Additional file 2: Appendix 4. A summary of the characteristics of all included studies. Appendix 5. A table summarising all of the randomised controlled trials that compared a quantitative pain score between intervention and control groups, with relevant results. Appendix 6. A table summarising the single cohort study that compared a quantitative pain score between intervention and control groups, with relevant results. Appendix 7. A table summarising all of the randomised controlled trials that compared post-procedural opioid requirements between intervention and control groups, with relevant results. Appendix 8. A table summarising the cohort studies that compared post-procedural opioid requirements between intervention and control groups, with relevant results. Appendix 9. A table summarising all of the randomised controlled trials that compared the length of hospital stay between intervention and control groups, with relevant results. Appendix 10. A table of the cohort studies that compared the length of hospital stay between intervention and control groups, with relevant results.

\section{Abbreviations}

AHRQ: Agency for Healthcare Research and Quality; Cl: Confidence Interval; HCC: Hepatocellular Carcinoma; IV: Intravenous; MCSD: Minimum Clinically Significant Difference; NOS: Newcastle-Ottawa Scale; NRS: Numeric Rating Scale; PCA: Patient Controlled Analgesia; ITT: Intention-to-treat; RCT: Randomised Controlled Trial; RoB 2: Risk of Bias 2; TACE: Trans-arterial chemoembolisation; UFE: Uterine Fibroid Embolisation; VAS: Visual Analogue Scale

\section{Acknowledgements}

Not applicable.

\section{Authors' contributions}

THS and HS were equally involved in the generation of the study concept and protocol, as well as the data extraction and analysis. THS and HS both equally contributed to drafting and revision of the manuscript. THS and HS both read and approved the manuscript.

\section{Funding}

This study was unfunded. There are no funding disclosures related to the conduct of this review.

\section{Availability of data and materials}

All data generated or analysed during this study are included in this published article [and its supplementary information files].

\section{Ethics approval and consent to participate}

Not applicable, not required for a systematic review.

\section{Consent for publication}

Not applicable.

\section{Competing interests}

The authors declare that they have no competing interests.

\section{Author details}

${ }^{1}$ Imperial College London, London, UK. ${ }^{2}$ Leeds Teaching Hospitals NHS Trust, Leeds, West Yorkshire, UK. 
Received: 2 October 2020 Accepted: 14 December 2020

Published online: 05 January 2021

\section{References}

Abusedera MA, Arafa UA, Ali EM (2014) Transcatheter administration of buffered Lidocaine for pain relief due to transarterial chemoembolization for HCC. Egypt J Radiol Nucl Med 45(2):403-408. https://doi.org/10.1016/j.ejrnm.2014. 03.005

Agency for Healthcare Research and Quality (2014) Newcastle-Ottawa Quality Assessment Form for Cohort Studies. https://www.ncbi.nlm.nih.gov/books/ NBK115843/bin/appe-fm3.pdf. Accessed 9 Sep 2020

Beecham GB, Bansal P, Nessel TA et al (2020) Lidocaine. [Updated 2020 Jul 10]. In: StatPearls. StatPearls Publishing, Treasure Island Available from: https://www. ncbi.nIm.nih.gov/books/NBK539881/

Cibils LA (1976) Response of human uterine arteries to local anesthetics. Am J Obstet Gynecol 126(2):202-210. https://doi.org/10.1016/0002-9378(76)90276-3

Downie WW, Leatham PA, Rhind VM et al (1978) Studies with pain rating scales. Ann Rheum Dis 37(4):378-381. https://doi.org/10.1136/ard.37.4.378

Duvnjak S, Andersen PE (2020) Intra-arterial lidocaine administration during uterine fibroid embolization to reduce the immediate postoperative pain: a prospective randomized study. CVIR Endovasc 3(1):10. https://doi.org/10. 1186/s42155-020-0099-4

Edwards RD, Moss JG, Lumsden MA et al (2007) Uterine-artery embolization versus surgery for symptomatic uterine fibroids. N Engl J Med 356(4):360370. https://doi.org/10.1056/NEJMoa062003

Hartnell GG, Gates J, Stuart K et al (1999) Hepatic chemoembolization: effect of intraarterial lidocaine on pain and postprocedure recovery. Cardiovasc Intervent Radiol 22(4):293-297. https://doi.org/10.1007/s002709900391

Higgins J, Thomas J, Chandler J et al (2019) Cochrane Handbook for Systematic Reviews of Interventions version 6.0 (updated July 2019). Cochrane. https:// training.cochrane.org/handbook. Accessed 7 Sep 2020

Ishihara H, Shoichiro I, Niimi J et al (2015) Risk factors and prevention of guiding catheter-induced vasospasm in neuroendovascular treatment. Neurol Med Chir (Tokyo) 55(3):261-265. https://doi.org/10.2176/nmc.0a.2014-0268

Katsumori T, Miura H, Yoshikawa T et al (2020) Intra-arterial Lidocaine Administration for Anesthesia after uterine artery embolization with Trisacryl gelatin microspheres for leiomyoma. J Vasc Interv Radiol 31(1):114-120. https://doi.org/10.1016/j.jvir.2019.09.007

Keyoung JA, Levy EB, Roth AR et al (2001) Intraarterial lidocaine for pain control after uterine artery embolization for leiomyomata. J Vasc Interv Radiol 12(9): 1065-1069. https://doi.org/10.1016/s1051-0443(07)61592-9

Lee S, Hahn S, Park S (2001) Intraarterial lidocaine administration for relief of pain resulting from transarterial chemoembolization of hepatocellular carcinoma: its effectiveness and optimal timing of administration. Cardiovasc Intervent Radiol 24(6):368-371. https://doi.org/10.1007/s00270-001-0073-z

Leung DA, Goin JE, Sickles C et al (2001) Determinants of postembolization syndrome after hepatic chemoembolization. J Vasc Interv Radiol 12(3):321326. https://doi.org/10.1016/s1051-0443(07)61911-3

Mason MC, Massarweh NN, Salami A et al (2015) Post-embolization syndrome as an early predictor of overall survival after transarterial chemoembolization for hepatocellular carcinoma. HPB (Oxford) 17(12):1137-1144. https://doi.org/10. 1111/hpb.12487

Moher D, Liberati A, Tetzlaff J et al (2009) Preferred reporting items for systematic reviews and meta-analyses: the PRISMA statement. PLoS Med 6(7):e1000097. https://doi.org/10.1371/journal.pmed.1000097

Molgaard CP, Teitelbaum GP, Pentecost MJ et al (1990) Intraarteria administration of lidocaine for analgesia in hepatic chemoembolization. J Vasc Interv Radiol 1(1):81-85. https://doi.org/10.1016/s1051-0443(90)72508-0

Myles PS, Myles DB, Galagher W et al (2017) Measuring acute postoperative pain using the visual analog scale: the minimal clinically important difference and patient acceptable symptom state. Br J Anaesth 118(3):424-429. https://doi. org/10.1093/bja/aew466

Noel-Lamy M, Tan KT, Simons ME et al (2017) Intraarterial Lidocaine for pain control in uterine artery embolization: a prospective, randomized study. J Vasc Interv Radiol 28(1):16-22. https://doi.org/10.1016/j.jvir.2016.10.001

Patanwala AE, Duby J, Waters D et al (2007) Opioid conversions in acute care. Ann Pharmacother 41(2):255-266. https://doi.org/10.1345/aph.1H421

Pereira J, Lawlor P, Vigano A et al (2001) Equianalgesic dose ratios for opioids. A critical review and proposals for long-term dosing. J Pain Symptom Manag 22(2):672-687. https://doi.org/10.1016/s0885-3924(01)00294-9
Saibudeen A, Makris GC, Elzein A et al (2019) Pain management protocols during uterine fibroid embolisation: a systematic review of the evidence. Cardiovasc Intervent Radiol 42(12):1663-1677. https://doi.org/10.1007/ s00270-019-02327-1

Sinclair R, Eriksson AS, Gretzer C et al (1993) Inhibitory effects of amide local anaesthetics on stimulus-induced human leukocyte metabolic activation, LTB4 release and IL-1 secretion in vitro. Acta Anaesthesiol Scand 37(2):159165. https://doi.org/10.1111/j.1399-6576.1993.tb03693.x

Spencer EB, Stratil P, Mizones H (2013) Clinical and periprocedural pain management for uterine artery embolization. Semin Intervent Radiol 30(4): 354-363. https://doi.org/10.1055/s-0033-1359729

Sterne JAC, Savović J, Page MJ et al (2019) RoB 2: a revised tool for assessing risk of bias in randomised trials. BMJ 366:14898. https://doi.org/10.1136/bmj.l4898

Swegle JM, Logemann C (2006) Management of common opioid-induced adverse effects. Am Fam Physician 74(8):1347

Wells GA, Shea B, O'Connell D et al (2012) The Newcastle-Ottawa Scale (NOS) for assessing the quality of nonrandomised studies in metaanalyses. http://www.ohri.ca/programs/clinical_epidemiology/oxford.asp. Accessed 7 Sep 2020

Widrich WC, Singer RJ, Robbins AH (1977) The use of intra-arterial lidocaine to control pain due to aortofemoral arteriography. Radiology 124(1):37-41. https://doi.org/10.1148/124.1.37

Zeng Z, Lu J, Shu C et al (2015) A comparision of nalbuphine with morphine for analgesic effects and safety : meta-analysis of randomized controlled trials. Sci Rep 5. https://doi.org/10.1038/srep10927

Zhan S, Li Y, Wang G et al (2005) Effectiveness of intra-arterial anesthesia for uterine fibroid embolization using dilute lidocaine. Eur Radiol 15(8):17521756. https://doi.org/10.1007/s00330-005-2686-0

Zhao SZ, Chung F, Hanna DB et al (2004) Dose-response relationship between opioid use and adverse effects after ambulatory surgery. J Pain Symptom Manag 28(1):35-46. https://doi.org/10.1016/j.jpainsymman.2003.11.001

\section{Publisher's Note}

Springer Nature remains neutral with regard to jurisdictional claims in published maps and institutional affiliations.

\section{Submit your manuscript to a SpringerOpen ${ }^{\circ}$ journal and benefit from:}

- Convenient online submission

Rigorous peer review

- Open access: articles freely available online

- High visibility within the field

- Retaining the copyright to your article

Submit your next manuscript at $\boldsymbol{\nabla}$ springeropen.com 